\title{
Uma breve revisão sobre a utilização de abelhas como bioindicadores de contaminação ambiental: Ênfase na Apis mellifera $L$.
}

A brief review on the use of bees as bioindicators of environmental contamination: Emphasis on Apis mellifera $L$.

Irineu Ferreira da Silva Neto, Graduando/Farmácia - Faculdade de Medicina Estácio de Juazeiro do Norte. Av. Tenente Raimundo Rocha, $\mathrm{n}^{\circ}$ 515, Cidade Universitária - Juazeiro do Norte - CE. CEP:63048080. Email: yrineuferreira@gmail.com

Maria Beatriz Silva Duarte, Bacharel/Farmácia - Faculdade de Medicina Estácio de Juazeiro do Norte. Av. Tenente Raimundo Rocha, $\mathrm{n}^{\circ}$ 515, Cidade Universitária - Juazeiro do Norte - CE. CEP:63048080. Email: mbeatrizduartefarmacia@gmail.com

Maria Aparecida Dias Fernandes Canuto, Bacharel/Farmácia - Faculdade de Medicina Estácio de Juazeiro do Norte. Av. Tenente Raimundo Rocha, $n^{\circ}$ 515, Cidade Universitária - Juazeiro do Norte - CE. CEP:63048080. Email: cidinhadiasfernandes@gmail.com

Annalu Moreira Aguiar, Mestranda em Ciências da Saúde - Faculdade de Medicina Estácio de Juazeiro do Norte. Av. Tenente Raimundo Rocha, $\mathrm{n}^{\circ}$ 515, Cidade Universitária - Juazeiro do Norte - CE. CEP:63048080. Email: annalumaguiar@gmail.com

\section{RESUMO}

A antropização generalizada e várias atividades humanas estão degradando o meio ambiente com intensidade crescente. Dessa forma, diferentes espécies de animais foram recentemente consideradas como bioindicadores de contaminação ambiental, em função da sua capacidade indicativa. Assim, o estudo desenvolvido possui como principal objetivo a verificação da capacidade e do grau de utilização de abelhas (Apis mellifera L.) como bioindicadores de contaminação ambiental, através de uma revisão de literatura, nas seguintes bases de dados: SciELO, PubMed e Google Scholar, utilizando os Descritores em Ciências da Saúde (DeCS): Apis mellifera, Biomarcadores ambientais "Environmental Biomarkers", Toxicologia "Toxicology", e Poluentes ambientais "Environmental Pollutants", combinados pelo operador booleano "AND". O período de publicações delimitado pelo estudo compreende desde o ano de 2015 até setembro de 2020, utilizando dados encontrados no idioma português ou inglês. Foram incluídos estudos disponíveis na íntegra, obtidos através da opinião de especialistas, os quais foram publicados em periódicos renomados. De acordo com a avaliação dos estudos coletados, pode-se verificar a existência de um cenário favorável para a aplicação de abelhas enquanto bioindicadores ambientais, sendo um dos fatores essenciais a diversidade do material amostrado por este tipo de animal, uma vez que, o processo de amostragem realizado acaba sendo bastante significativo e representativo, porém, em contrapartida, ainda se faz bastante perceptível às fragilidades que este tipo de amostragem pode vir a apresentar. É válido salientar que o estudo prévio da espécie que será utilizada, assim como da região a qual se pretende analisar, é indispensável para que se tenha uma coleta de dados mais apurada.

Palavras-Chave: Meio Ambiente, Toxicologia, Poluente, Biomarcador.

\section{ABSTRACT}

Widespread anthropization and various human activities are degrading the environment with increasing intensity. In this way, different species of animals were studied as bioindicators of environmental contamination, depending on their indicative capacity. Thus, the developed study has as main objective the verification of the capacity and the degree of use of bees (Apis mellifera L.) as bioindicators of environmental contamination, through a literature review, in the following databases: SciELO, PubMed and Google Scholar, using the Health Sciences Descriptors (DeCS): Apis mellifera, Biomarcadores ambientais "Environmental Biomarkers", Toxicologia "Toxicology", e Poluentes ambientais "Environmental Pollutants", combinados pelo operador booleano "AND". The period of publications delimited by the study runs from 2015 to September 2020, using data found in Portuguese or English. Studies available in full, obtained through expert opinion, were included, which were published in renowned journals. According to the evaluation of the collected studies, it is possible to verify the existence of a favorable scenario for the application of bees as environmental bioindicators, one of the essential ones being the diversity of the material sampled by this type of animal, since, the process of The sampling carried out ends up being quite significant and representative, however, in contrast, it is still quite noticeable to the weaknesses that this type of sampling may come to present. It is worth noting that the previous study of the species that will be used, as well as the region to be analyzed, is indispensable for the collection of more accurate data.

Keywords: Environment, Toxicology, Pollutant, Biomarker 
NETO, Irineu Ferreira da Silva; DUARTE, Maria Beatriz Silva; CANUTO, Maria Aparecida Dias Fernandes; AGUIAR, Annalu Moreira (2021). Uma breve revisão sobre a utilização de abelhas como bioindicadores de contaminação ambiental: Ênfase na Apis mellifera L.

\section{Introdução}

A antropização generalizada e várias atividades humanas estão degradando o meio ambiente com intensidade crescente. A consciência dos danos incalculáveis, incluindo doenças graves, causados pelo despejo contínuo de produtos químicos no meio ambiente, cresceu consideravelmente nas últimas décadas, especialmente em relação aos poluentes persistentes e acumulativos. No entanto, o número de agentes tóxicos liberados neste meio é, atualmente, muito expressivo e apenas uma pequena fração dessas substâncias é monitorada regularmente. Dessa forma, as diretivas ambientais reduziram os níveis permitidos de poluentes na água, no ar, nos alimentos e aumentaram a lista de produtos químicos que são proibidos e precisam ser avaliados (GIROTTI et al., 2020).

Para avaliar a contaminação ambiental, utiliza-se a bioindicação, como um registro sensível e dependente de fatores ambientais alterados antropogênicos que tem dimensões distintas de sistemas/objetos biológicos sob circunstâncias definidas. Enquanto isso, a presença de populações bioindicadoras, ou estrutura populacional, reflete a saúde do meio ambiente e pode ser considerada altamente informativa. No entanto, a funcionalidade dos indivíduos e a caracterização de sua integridade fisiológica requerem ferramentas que possam atuar como biomarcadores de exposição a estressores ambientais (MILONE et al., 2021; DAVODPOUR et al., 2019).

A banalidade da contaminação, mesmo a distâncias consideráveis de centros industriais e áreas econômicas intensamente utilizadas, tem-se tornado o interesse de muitos pesquisadores. A questão de avaliar o estado do meio ambiente com a ajuda de organismos vivos tornou-se uma parte muito importante no controle do meio ambiente (SKORBIŁOWICZ; SKORBIŁOWICZ; CIEŚLUK, 2018).

Ao se avaliar as características principais dos estudos clássicos existentes, percebe-se que a maioria dos métodos de monitoramento empregados no desenvolvimento dos trabalhos requerem equipamentos caros ou cobre apenas uma pequena área de interesse. E, nesse contexto, diferentes espécies de plantas foram propostas como bioindicadores de poluição ambiental, porém, os vegetais são organismos estacionários e conseguem mensurar apenas as proximidades da área em que se localizam. Portanto, em função de uma maior capacidade indicativa, diferentes espécies de animais foram recentemente consideradas como bioindicadores (MILONE et al., 2021; NGAT et al., 2020; BOTTEON, 2016).

Nesse sentido, as abelhas (Apis mellifera L.) com amplo comportamento de forrageamento podem absorver poluentes de todos os compartimentos ambientais, incluindo solo, néctar e água, o que faz com que a espécie seja considerada potencialmente útil em um nível elevado como bioindicadora detecção e monitoramento da poluição ambiental (DAVODPOUR et al., 2019).

Dessa forma, o estudo desenvolvido possui como principal objetivo a verificação da capacidade e do grau de utilização de abelhas (Apis mellifera L.) como bioindicadores de contaminação ambiental, através de um estudo de revisão da literatura. 
NETO, Irineu Ferreira da Silva; DUARTE, Maria Beatriz Silva; CANUTO, Maria Aparecida Dias Fernandes; AGUIAR, Annalu Moreira (2021). Uma breve revisão sobre a utilização de abelhas como bioindicadores de contaminação ambiental: Ênfase na Apis mellifera L.

\section{Material e Métodos}

O trabalho caracteriza-se como um estudo de revisão de literatura do tipo integrativa, onde se realizou a coleta de arquivos nas seguintes bases de dados: SciELO (Scientific Electronic Library Online), PubMed (National Library of Medicine) e Google Scholar, utilizando os Descritores em Ciências da Saúde (DeCS): Apis mellifera, Biomarcadores ambientais "Environmental Biomarkers", Toxicologia "Toxicology", e Poluentes ambientais "Environmental Pollutants", combinados pelo operador booleano "AND".

O período de publicações delimitado pelo estudo compreende desde o ano de 2015 até setembro de 2020, utilizando dados encontrados no idioma português ou inglês. Foram incluídos estudos disponíveis na íntegra, obtidos através da opinião de especialistas, os quais foram publicados em periódicos renomados. Os estudos selecionados apresentavam pelo menos dois dos descritores supracitados, com o conteúdo relativo ao objetivo do estudo, partindo sempre do foco central desta pesquisa, ou seja, aqueles que apresentavam indícios sobre a utilização de abelhas como bioindicadores de contaminação ambiental. Em contrapartida, estudos incompletos, que não se enquadravam dentro do período temporal delimitado pela pesquisa, linguagens selecionadas, ou não apresentam os descritores supracitados, foram excluídos.

A partir da busca realizada nas bases de dados, foram encontrados 120 estudos, utilizando a combinação dos descritores. No processo de seleção, os artigos foram avaliados inicialmente por meio da leitura dos títulos e resumos. Em seguida, foram aplicados os critérios de inclusão e exclusão. Para a síntese desta revisão de literatura, utilizou-se um total de 12 estudos.

\section{Resultados e Discussão}

A deposição de elementos (tóxicos e não tóxicos) no meio ambiente, principalmente em plantas é uma das preocupações mais urgentes na ciência da segurança e na qualidade dos alimentos. Estudos mostram o impacto de diferentes elementos e substâncias tóxicas a saúde humana, bem como os mecanismos de absorção e bioacumulação. Por exemplo, alguns elementos metálicos (chumbo, cádmio, mercúrio e arsênico), e vários tipos de Material Particulado (MP) são considerados cancerígenos em potencial e estão associados à etiologia de uma série de doenças internas (ĆIRIĆ et al., 2020).

Ao longo dos anos, várias doenças humanas têm sido associadas à exposição ao MP, que pode ser responsável por efeitos cumulativos na saúde a curtos e longos prazos. Mortalidade precoce neonatal, morbidade, doenças cardiovasculares e cardiopulmonares, asma e câncer de pulmão estão entre os efeitos mais frequentes observados em pacientes expostos a partículas transportadas pelo ar. Pesquisas toxicológicas têm demonstrado que, em nível celular, o MP pode induzir citotoxicidade, neurotoxicidade, mutagenicidade, estimulação de fatores pró-inflamatórios e até mesmo alterações epigenéticas do DNA com consequências na expressão gênica (NEGRI et al., 2015).

A situação qualitativa do meio ambiente e dos contaminantes ali encontrados é sinalizada por um conjunto de indicadores, conhecidos como bioindicadores, vários dos quais são responsáveis por mostrar impactos progressivos de diferentes tipos de poluentes. Tendo abordado a influência de 
NETO, Irineu Ferreira da Silva; DUARTE, Maria Beatriz Silva; CANUTO, Maria Aparecida Dias Fernandes; AGUIAR, Annalu Moreira (2021). Uma breve revisão sobre a utilização de abelhas como bioindicadores de contaminação ambiental: Ênfase na Apis mellifera L.

vários bioindicadores na poluição ambiental, foi revelado que os bioindicadores são sensíveis a qualquer perturbação em qualquer ambiente. No que diz respeito à poluição, a qualidade de um ecossistema pode ser avaliada por um organismo, que na verdade é um indicador e desempenha um papel fundamental no monitoramento de suas mudanças (ASIF; MALIK; CHAUDHRY, 2018).

Nesse contexto, as abelhas são um meio altamente eficiente de realizar pesquisas para o monitoramento ambiental (fazendo inúmeras inspeções por dia), e a maioria de seus voos possuem uma considerável distância de sua colônia. Elas visitam quase todos os setores ambientais (solo, vegetação, água e ar) em uma área circular com um raio de aproximadamente $12 \mathrm{~km}$ ao redor da colmeia, fornecendo inúmeras amostras por meio de critérios específicos para cada tipo de abelha. Dessa forma, reúnem néctar, pólen, própolis e água, todos os quais são trazidos para a colmeia e armazenados de acordo com critérios verificáveis. Uma vez na colmeia, esses produtos são fáceis de amostrar e, subsequentemente, analisar quanto a contaminantes, ou seja, as abelhas agem como bioacumuladores (DONKERSLEY, 2019; GIROTTI et al., 2020).

Dessa forma, aos poucos foram sendo elaborados protocolos de monitoramento utilizando abelhas como bioindicadores, os quais servem como parâmetros na avaliação de contaminação ambiental, como pode ser visualizado no Quadro 1.

Quadro 1. Protocolos de monitoramento de contaminantes ambientais utilizando abelhas como bioindicadores.

\begin{tabular}{|c|c|c|c|c|}
\hline Poluente & Matriz analítica & Método empregado & Dados suplementares & Autor/ano \\
\hline Pesticidas & $\begin{array}{c}\text { Abelhas mortas } \\
\text { coletadas } \\
\text { semanalmente } \\
\text { no solo }\end{array}$ & SPME, GC, HPLC & $\begin{array}{c}\text { Limite de } \\
\text { mortalidade: } 250 \\
\text { abelhas / semana / } \\
\text { estação / Mapas de } \\
\text { cultivo de plantas / } \\
\text { Identificação de pólen }\end{array}$ & \multirow{3}{*}{$\begin{array}{l}\text { GIROTTI et } \\
\text { al., } 2020\end{array}$} \\
\hline Metais pesados & $\begin{array}{c}\text { Abelhas } \\
\text { forrageiras } \\
\text { capturadas a } \\
\text { cada } 15 \text { dias, e } \\
\text { mel fresco } \\
\text { coletado } \\
\text { mensalmente }\end{array}$ & $\begin{array}{c}\text { Mineralização - } \\
\text { identificação de metais em } \\
\text { amostras contendo abelhas } \\
\text { vivas ou mortas, } \\
\text { espectrometria de absorção } \\
\text { atômica de chama e } \\
\text { eletroquímica }\end{array}$ & $\begin{array}{l}\text { Monitoramento das } \\
\text { condições ambientais } \\
\text { / Identificação de } \\
\text { pólen (análise } \\
\text { palinológica) }\end{array}$ & \\
\hline Radionuclídeos & $\begin{array}{c}\text { Abelhas mortas } \\
\text { coletadas } \\
\text { semanalmente } \\
\text { no solo }\end{array}$ & $\begin{array}{l}\text { Espectrometria gama de } \\
\text { cristal de germânio }\end{array}$ & $\begin{array}{l}\text { Monitoramento das } \\
\text { condições ambientais } \\
\text { / Identificação de } \\
\text { pólen (análise } \\
\text { palinológica) }\end{array}$ & \\
\hline
\end{tabular}

Fonte: dados da pesquisa, 2020.

A maioria das substâncias não são transferidas do solo para as abelhas diretamente pela absorção das plantas, mas sim pela erosão da camada superficial do solo e deposição de partículas atmosféricas nas flores e, consequentemente, no pólen. Ao beber água ou coletar pólen e néctar, as abelhas também são expostas aos contaminantes presentes nelas. Além disso, o contato das abelhas com as partes da planta ou solo que apresentam deposição de material particulado atmosférico pode fazer com que essas partículas venham a aderir aos seus corpos peludos. Tendo em vista que os 
NETO, Irineu Ferreira da Silva; DUARTE, Maria Beatriz Silva; CANUTO, Maria Aparecida Dias Fernandes; AGUIAR, Annalu Moreira (2021). Uma breve revisão sobre a utilização de abelhas como bioindicadores de contaminação ambiental: Ênfase na Apis mellifera L.

agentes tóxicos, geralmente, não causam a mortalidade das abelhas, estas foram propostas como bioindicadores adequados da poluição (ZARIĆ et al., 2017).

Portanto, as amostras de poluentes que ficam contidas nas abelhas representam a poluição presente no ar, água e solo. Estas vem sendo cada vez mais utilizadas como bioindicadores nas últimas décadas, principalmente para detectar poluição por metais em diferentes ambientes, incluindo regiões urbanas, rurais, áreas industriais e áreas protegidas (ZARIC et al., 2018; BOTTEON, 2016)

Uma das preocupações encontradas pelos pesquisadores para utilização de abelhas como bioindicadores é que os pesticidas usados na agricultura (especialmente na primavera e no verão, quando as atividades agrícolas atingem seu pico) podem ser a causa da mortalidade em grande escala das abelhas, além disso, também podem acabar ocasionando contaminação em produtos apícolas. Ou seja, a presença de xenobióticos pode prejudicar o monitoramento, bem como a qualidade dos produtos, colocando assim em risco a saúde humana (MILONE et al., 2021; BÖHME, et al., 2018; BARGAŃSKA; ŚLEBIODA; NAMIEŚNIK, 2016).

As abelhas, devido ao seu amplo comportamento de forrageamento, apresentam grande potencial para o monitoramento da qualidade ambiental. Nesse contexto, Davodpour et al., (2019) realizaram um estudo o qual objetivou utilizar as abelhas como bioindicadores para a detecção de metais tóxicos e essenciais ( $\mathrm{Cd}, \mathrm{Cr}$, Cu e Fe) no ambiente. No total, 180 amostras de solo, planta (incluindo raiz e parte aérea), abelhas e mel foram coletados de 12 locais nas principais regiões de apicultura e produção de mel na província de Markazi, Irã em 2016. Após a digestão ácida das amostras, as concentrações de metal foram medidas por espectrômetros de emissão óptica de plasma indutivamente acoplados, onde os resultados indicaram que as abelhas podem ser usadas para detectar os padrões espaciais de contaminação por metais no ambiente em que vivem, sugerindo assim a sua utilização em outros setores.

Já no estudo realizado por Zhou et al., (2018) foram comparados os dados das abelhas nativas da Austrália (Tetragonula carbonaria) com as amostras correspondentes da abelha europeia mais comum (Apis melifera L.). Embora as composições isotópicas fossem semelhantes em ambas às espécies, diferenças significativas nas concentrações ficaram evidentes no conjunto de oligoelementos, tanto nas abelhas quanto em seus produtos. A associação estatística entre T. carbonaria e concentrações de contaminantes ambientais co-localizados foi mais forte e predominante do que aquelas em abelhas europeias, o que pode ser atribuído à sua menor distância de forrageamento $(0,3-0,7 \mathrm{~km}$ versus $5-9 \mathrm{~km}$, respectivamente). Isso implica que T. carbonaria pode ser mais adequada para avaliar pequenas variações em escala espacial de concentrações de oligoelementos do que as abelhas europeias, o que expõe a necessidade de se estudar especificamente cada espécie, bem como o ambiente que estas se encontram (ZHOU et al., 2018).

Apesar do conhecido papel das abelhas no monitoramento ambiental, estudos utilizando estes himenópteros como amostradores ativos de material particulado aerotransportado são completamente carentes, mesmo que a descrição morfológica e a caracterização físico-química do MP coletado pelas abelhas forneçam informações precisas sobre as fontes de emissão e os riscos potenciais à saúde. Na verdade, este é um ponto chave para o desenvolvimento de estratégias de 
NETO, Irineu Ferreira da Silva; DUARTE, Maria Beatriz Silva; CANUTO, Maria Aparecida Dias Fernandes; AGUIAR, Annalu Moreira (2021). Uma breve revisão sobre a utilização de abelhas como bioindicadores de contaminação ambiental: Ênfase na Apis mellifera L.

controle adequadas, a fim de reduzir o impacto dos poluentes no meio ambiente e na saúde pública (NUNES-SILVA et al., 2019).

Uma forma confiável e econômica de avaliar as mudanças no meio ambiente é possível por meio de espécies indicadoras, mas a seleção de um indicador específico representa um verdadeiro desafio, seguido de sua identificação, bem como da relação entre os indicadores e suas aplicações particulares. Como resultado, os indicadores ambientais, ecológicos e de biodiversidade cumprem seu objetivo de monitorar a qualidade ambiental. A situação atual, em escala global, requer bioindicadores de baixo custo e que possam proporcionar confiabilidade em sua detecção, além disso, os mesmos servirão para mitigar os impactos da poluição sobre o meio ambiente (ASIF; MALIK; CHAUDHRY, 2018).

\section{Conclusões}

De acordo com a avaliação dos estudos coletados, pode-se verificar a existência de um cenário favorável para a aplicação de abelhas enquanto bioindicadores ambientais, especialmente pela abrangência de materiais amostrados por este tipo de animal, uma vez que, o processo realizado acaba sendo bastante significativo e representativo. Porém, se faz bastante perceptível às fragilidades que este tipo de amostragem pode vir a apresentar, principalmente ao se considerar os interferentes, naturais ou não, que acabam comprometendo tanto a qualidade, quanto a integridade dos resultados obtidos.

As formas de aplicação metodológica também não apresentam uma robustez concretizada, o que acaba sendo compreendido pelo fato de não existirem protocolos bem determinados que possam orientar o desenvolvimento significativo do trabalho.

As abelhas definitivamente apresentam uma capacidade amostral superior a outros tipos de bioindicadores, porém a Apis mellifera L., em especial, não vem a ser uma das melhores escolhas para o desenvolvimento deste tipo de trabalho no que se refere a abelhas, tendo em vista que existem outras espécies que possuem uma melhor e maior capacidade amostral, gerando, portanto, resultados mais representativos do que a própria Apis melífera $\mathrm{L}$.

É válido salientar que o estudo prévio da espécie que será utilizada, assim como da região a qual se pretende analisar, é indispensável para que se tenha uma coleta de dados mais apurados e que possam ser tratados de maneira mais eficiente, vindo assim a garantir ao estudo uma maior relevância, em função da qualidade dos dados obtidos.

\section{Referências}

ASIF, Nayyab; MALIK, Muhammad; CHAUDHRY, F. N. A review of on environmental pollution bioindicators. Pollution, v. 4, n. 1, p. 111-118, 2018.

BARGAŃSKA, Żaneta; ŚLEBIODA, Marek; NAMIEŚNIK, Jacek. Honey bees and their products: Bioindicators of environmental contamination. Critical Reviews in Environmental Science and Technology, v. 46, n. 3, p. 235-248, 2016.

BÖHME, Franziska et al. From field to food-will pesticide-contaminated pollen diet lead to a 
NETO, Irineu Ferreira da Silva; DUARTE, Maria Beatriz Silva; CANUTO, Maria Aparecida Dias Fernandes; AGUIAR, Annalu Moreira (2021). Uma breve revisão sobre a utilização de abelhas como bioindicadores de contaminação ambiental: Ênfase na Apis mellifera $L$.

contamination of royal jelly?.Apidologie, v. 49, p. 112-119, 2018.

BOTTEON Victor Wilson. Perspectivas de Uso de Insetos Bioindicadores Ambientais em Trabalhos Periciais. Brazilian Journal of Forensic Sciences, Medical Law and Bioethics, v. 5, n. 4, p. 383-401, 2016.

ĆIRIĆ, Jelena et al. Honey Bees and Their Products as Indicators of Environmental Element Deposition. Biological Trace Element Research, p. 1-8, 2020.

DAVODPOUR, Rezvan et al. Honeybees (Apis mellifera L.) as a potential bioindicator for detection of toxic and essential elements in the environment (case study: Markazi Province, Iran). Archives of environmental contamination and toxicology, v. 77, n. 3, p. 344-358, 2019.

DONKERSLEY, Philip. Trees for bees. Agriculture, ecosystems \& environment, v. 270, p. 79-83, 2019.

GIROTTI, Stefano et al. Bioindicators and biomonitoring: honeybees and hive products as pollution impact assessment tools for the Mediterranean area. Euro-Mediterranean Journal for Environmental Integration, v. 5, n. 3, p. 1-16, 2020.

MILONE, Joseph P. et al. Colony-level pesticide exposure affects honey bee (Apis mellifera L.) royal jelly production and nutritional composition. Chemosphere, v. 263, p. 128183, 2021.

NEGRI, Ilaria et al. Honey bees (Apis mellifera, L.) as active samplers of airborne particulate matter. PLoS One, v. 10, n. 7, p. 0132491, 2015.

NGAT, Tran Thi et al. Honeybee products as potential bioindicators of heavy metals contamination from Northern Vietnam. Vietnam Journal of Biotechnology, v. 18, n. 2, p. 373-384, 2020.

NUNES-SILVA, P. et al. Applications of RFID technology on the study of bees. Insectes sociaux, v. 66, n. 1, p. 15-24, 2019.

ZARIĆ, Nenad M. et al. Use of honeybees (Apis mellifera L.) as bioindicators for assessment and source appointment of metal pollution. Environmental Science and Pollution Research, v. 24, n. 33, p. 25828-25838, 2017.

ZARIC, Nenad M. et al. Avaliação das variações espaciais e temporais nas concentrações de elementos traço usando abelhas (Apis mellifera) como bioindicadores. PeerJ, v. 6, p. 5197, 2018.

ZHOU, Xiaoteng et al. Identifying sources of environmental contamination in European honey bees (Apis mellifera) using trace elements and lead isotopic compositions. Environmental science \& technology, v. 52, n. 3, p. 991-1001, 2018. 